\title{
A prototype for the on-line monitoring system for nuclear waste storage
}

\author{
Alfio Pappalardo ${ }^{1}$ \\ INFN-Laboratori Nazionali del Sud, Via Santa Sofia 62, Catania Italy \\ E-mail: a.pappalardo@lns.infn.it
}

\section{Luigi Cosentino}

INFN-Laboratori Nazionali del Sud, Via Santa Sofia 62, Catania Italy

E-mail: cosentinollns.infn.it

\section{Paolo Finocchiaro}

INFN-Laboratori Nazionali del Sud, Via Santa Sofia 62, Catania Italy

E-mail: finocchiarodlns.infn.it

\section{Massimo Piscopo}

INFN-Laboratori Nazionali del Sud, Via Santa Sofia 62, Catania Italy

E-mail: piscopollns.infn.it

\section{Carlotta Scirè Scappuzzo}

Ansaldo Nucleare Via Giosue' Carducci, 10, 00187 Roma, Italy

E-mail: scireelns.infn.it

\section{Sergio Scirè Scappuzzo}

Ansaldo Nucleare Via Giosue' Carducci, 10, 00187 Roma, Italy

E-mail: scirecelns.infn.it

\begin{abstract}
We propose a system for the on-line monitoring of short and medium term radioactive waste repositories. Such a system is distributed, fine-grained, robust, reliable, and must be based on low-cost components. It could, in principle, open new perspectives on the modality of waste packaging and storage. In particular we propose to employ a new family of cheap and powerful micro sensors to be placed in shape of a fine grid around each single drum.
\end{abstract}

XLIX International Winter Meeting on Nuclear Physics- BORMIO2011

Bormio, Italy

January 24-28 2011

\footnotetext{
$1 \quad$ Speaker
} 


\section{Introduction}

Among the many issues related to the disposal of short and medium term radioactive waste, there is the storage inside suitable sites where a high level of safety must be guaranteed. In order to detect possible leaks of radioactive material and to efficiently reduce the risks of contamination for the operators and for the environment, the IAEA suggests the possible adoption of real time monitoring systems [1]. At the moment such a kind of system does not seem to be in operation in any of the current storage sites in the world. The monitoring is done periodically by means of robots or operators, plus some overall monitoring of fixed locations inside the repository [2].

The DMNR project (Detector Mesh for Nuclear Repositories) has started recently to study the possibility of an integrated solution for on-line monitoring of the radiation field, providing a 3-dimensional map of the radioactivity produced by the waste. This way a possible radioactive leak from a waste drum, could be recognized by means of an increase of the local activity. This could be achieved by means of traditional Geiger-Müller counters, but the cost of such a solution would be prohibitive. Therefore we have suggested to adopt a mesh of low cost radiation detectors, namely scintillating fibres, arranged as a grid of sensors around each waste drum (Figure 1). These allow to record continuously the measured activity, in order to check the instant rate and also the counting history around each drum. It is also foreseen the installation of a number of more conventional reference detectors in prefixed locations.

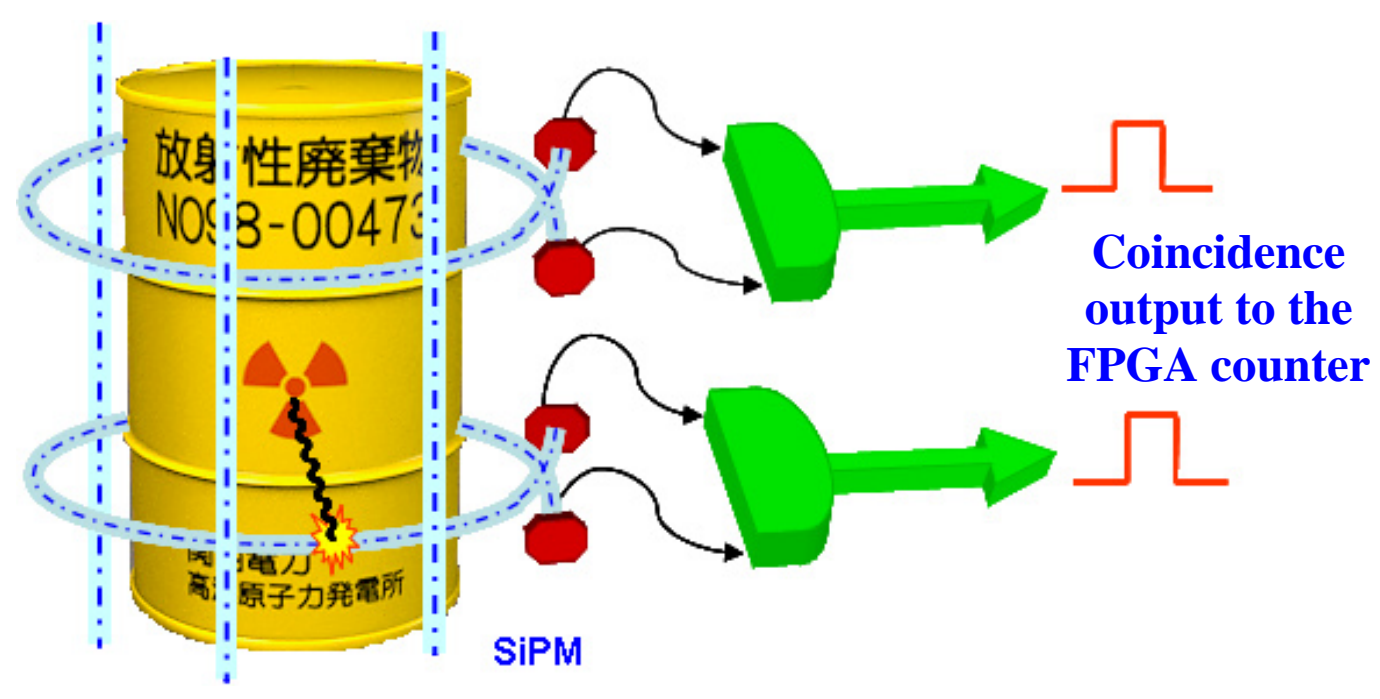

Figure.1 A possible layout of the fibres around the drum.

\section{Geant simulation for gamma rays}

The gamma rays of interest to DMNR project fall in the range 0.1 to $2.5 \mathrm{MeV}$ and interact with detector and absorbers by three major processes: photoelectric absorption, Compton scattering, and pair production [3].

In the photoelectric absorption process, the gamma ray may interact with a bound atomic electron in such a way that it loses all of its energy and ceases to exist as a gamma ray. Some of 
the gamma-ray energy is used to overcome the electron binding energy, whereas the remainder is transferred to the freed electron as kinetic energy. The probability of photoelectric absorption depends on the gamma-ray energy, the electron binding energy, and the atomic number of the atom.

Compton scattering is the process whereby a gamma ray interacts with a free or weakly bound electron $\left(E_{\gamma}>E_{b}\right)$ transferring to it part of its energy. The electron becomes a free electron with kinetic energy equal to the difference of the energy lost by the gamma ray and the electron binding energy. Because the electron binding energy is very small compared to the gamma-ray energy, the kinetic energy of the electron is very nearly equal to the energy lost by the gamma ray.

In pair production a gamma ray with an energy of at least $1.022 \mathrm{MeV}$ can create an electron-positron pair when it is under the influence of the strong electromagnetic field in the vicinity of a nucleus. If the gamma ray energy exceeds $1.022 \mathrm{MeV}$, the excess energy is shared between the electron and positron as kinetic energy. The electron and positron from pair production are rapidly slowed down in the absorber. After losing its kinetic energy, the positron combines with an electron in an annihilation process, which releases two gamma rays with energies of $0.511 \mathrm{MeV}$. The probability of pair production varies approximately as the square of the atomic number $\mathrm{Z}$ and is significant in high- $\mathrm{Z}$ elements. The produced electrons, in the three processes described above, release their energy to the scintillating medium producing optical photons.

In order to evaluate the scintillating fibre response to the gamma rays, and to choose the most appropriate geometry of the detector mesh and the number of fibres to be placed around each drum as a trade off between the efficiency of the overall system and its cost, we have carried out several simulations based on Geant Monte Carlo code (Geant3.21)([4]).

The simulations were performed for a plastic scintillating fibre (polyvinyl-toluene by Pol.Hi.Tec) $1.2 \mathrm{~m}$ long and $1 \mathrm{~mm}$ diameter. The considered sources were a point-like piece of ${ }^{60} \mathrm{Co}$ and ${ }^{137} \mathrm{Cs}$.

\section{$120 \mathrm{~cm}$}

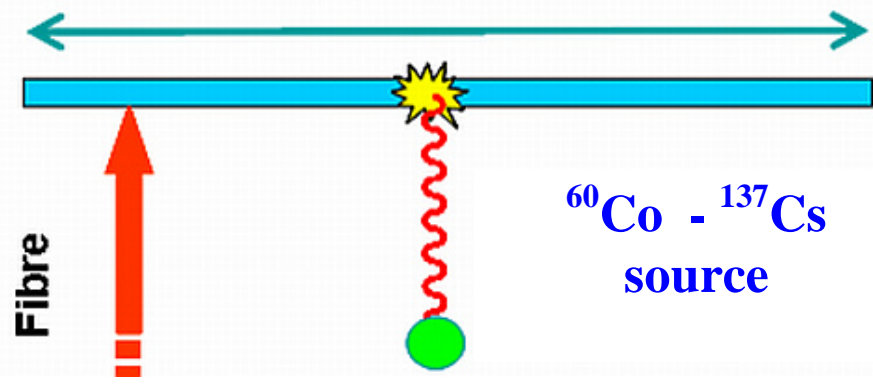

Figure.2 Basic scheme of the simulated geometry.

In our first simulation with one of the sources was placed at several distances from the center of the fiber, and we took into account the two gamma rays and the beta particle coming from the decay produced by the ${ }^{60} \mathrm{Co}$ decay $(1.17 \mathrm{MeV}$ and $1.33 \mathrm{MeV})$. We performed several runs with source-fibre distance variable from $3 \mathrm{~mm}$ to $20 \mathrm{~mm}$, considering only those events producing a detectable light signal at both fibre ends in order to evaluate the fibre efficiency. 
Under the same geometrical conditions we estimated the fibre efficiency to ${ }^{137} \mathrm{Cs}(662 \mathrm{KeV})$ point-like source.

After that, in the same geometrical conditions we had conducted experimental tests to estimate the intrinsic efficiency of the fibers, employing the source of ${ }^{60} \mathrm{Co}$ and ${ }^{137} \mathrm{Cs}$ in order to validate the Geant simulations. The measurements were carried out by coupling each end of the fiber with two silicon photomultiplier sensors, namely Hamamatsu MPPC S10362-11-100U, biased at 70V. The evaluated and measure fiber efficiency is reported in figure 3. Unfortunately these sensors are strongly affected by the presence of correlated noise, i.e. cross-talk, (see figure 4 and refs. [10]), and forced us to set a high value of threshold (6 photons). In the meantime we plan to set up new measurements using better performing silicon photomultipliers biased at $30 \mathrm{~V}$, produced by STMicroelectronics, under more realistic conditions.

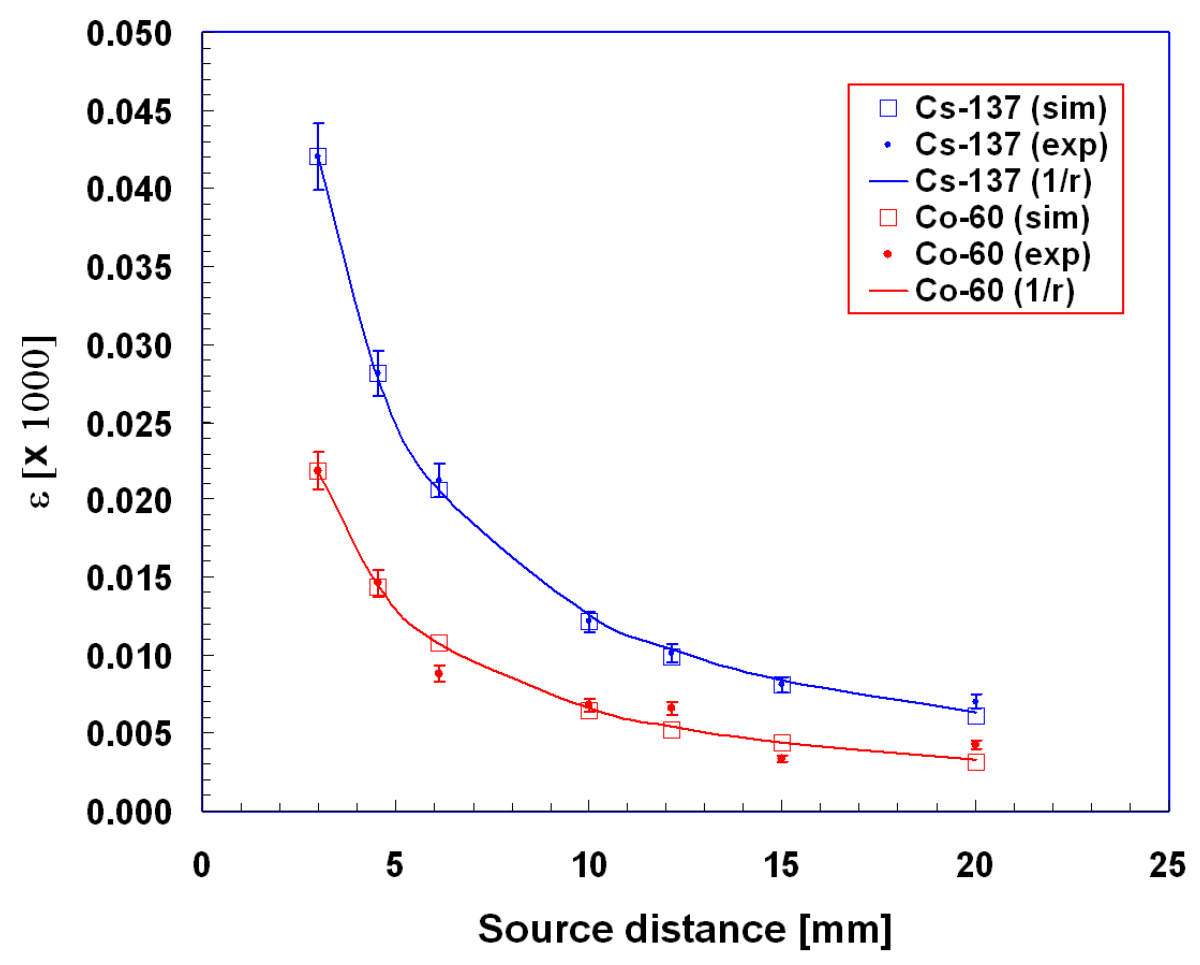

Figure.3 Plot of the measured and simulated intrinsic efficiency for a plastic scintillating fiber employing $a^{60}$ Co and ${ }^{137}$ Cs point sources.

\section{The experimental apparatus}

The mesh of detectors has to be properly distributed, fine-grained, robust, reliable, and should be based on low-cost components. In addition, the sensors have to be operated in Geiger mode (on/off), since they are just needed to count the ionizing radiation events.

Each detector consists of a scintillating optical fibre, 1-2 $\mathrm{m}$ long. The fibre is a plastic one made of a scintillating core of polystyrene and a PMMA cladding, manufactured by SaintGobain Crystals. It has a $1 \mathrm{~mm}$ diameter with a $40 \mu \mathrm{m}$ thick cladding and an attenuation length $\lambda_{\mathrm{at}}=3.3$ meter. Its minimum trapping efficiency for isotropic light emission is $3.4 \%$ at each fibre end, as can be easily calculated starting from the refraction index values $n_{1}=1.60$ (core) 
and $\mathrm{n}_{2}=1.48$ (cladding) [5] [6] [7]. The decay time of the scintillation light, $\tau \sim 3 \mathrm{~ns}$, allows to measure activities of the order of $\mathrm{MHz}$ (the expected count rate is in the range $10^{3}-10^{5} / \mathrm{fibre} / \mathrm{s}$ ). The scintillation spectrum lies in the green region with a maximum around $520 \mathrm{~nm}$.

From the point of view of radiation hardness, even though it is low for this kind of fibres, as the overall efficiency is low, the damage will be kept low as well. A reference drum contains 20 liters of waste incorporated in concrete and surrounded by a layer of mortar, with a total activity of $\sim 10^{12} \mathrm{gamma} / \mathrm{s}$ corresponding to a dose rate of $10-100 \mathrm{mGy} / \mathrm{h}$. Under these assumptions we estimated, for a detector placed close to a drum, a life of the order of at least 100 years [3].

The fibres are arranged around each drum in longitudinal and/or ring geometry, as in figure 1. Both geometries can be adopted, as the most likely leak position has to be determined. Each fibre can intercept radiation coming out of the drum wall, mostly gamma rays, so that the energy released inside its active volume is converted into scintillation light, that propagates to both ends of the fibre itself. The light yield of these fibres is of the order of $10^{4}$ photons $/ \mathrm{MeV}$. Considering that: (i) the expected energy range of the gamma rays is in the range $0.1-2.5$ $\mathrm{MeV}$; (ii) the average energy released in a fibre is roughly of the order of $100 \mathrm{keV}$; (iii) at least roughly $3 \%$ of the produced scintillation light is collected at each fibre end (also considering the attenuation); as a consequence we have that the number of photons to be detected can be as low as 2-30.

Using standard photomultiplier tubes would pose issues like cost, non-ruggedness, fragility, high-voltage power supply, damage from accidental exposure to ambient light. Therefore we need a rugged photodetector to be coupled to each fibre end, with a sensitivity down to the single photon and capable of fast timing, in order to select events in strict coincidence between the two fibre sides (figure 1). When a pulse is detected on both sensors within few tens of nanoseconds, it is recognized as an ionising event, counted and recorded to compute the overall activity.

\subsection{The SiPM photosensor}

The high sensitivity photodetector we have decided to use is the newly born SiPM (Silicon PhotoMultiplier), a promising cheap detector that is reliable and easy to handle. It is a 2dimensional array of a Single Photon Avalanche Diode (SPAD) cells. A SPAD is a p-n junction operating in Geiger mode (on/off) at low voltage $(\approx 30$ to 70 Volts depending on the manufacturer) and made up in silicon planar technology. The junction is biased a little above the breakdown voltage $(\approx 10 \%$ of the breakdown voltage itself), and it remains inactive until a photon is absorbed in the depletion region. The outputs of all cells are connected together to form one common signal. Each SPAD operates in the Geiger regime, basically producing the same pulse (rise time $-\approx 0.5-5 \mathrm{~ns}$ ) when fired by a photon or because of thermal generation. When a fast light pulse is detected by the SIPM, its output is the sum of the identical pulses produced by each fired pixel. Its sensitivity can be as low as the single photon, but such a lower limit is strongly affected by dark noise. It is also affected by cross-talk, each time a cell is triggered the electrons in the avalanche can themselves reach the neighbouring cells and trigger 
them. The cross-talk effect can be put into evidence by plotting the dark noise rate versus the threshold, expressed in number of equivalent pixels (figure 4).

One would expect the dark noise dropping when the threshold is set above 1 pixel, since it is unlikely that two uncorrelated pixels fire simultaneously.

\subsection{The SiPM features}

In this perspective we have tested four SiPM models, produced by Hamamatsu photonics, SensL, FBK-IRST, and STMicroelectronics (not yet available on the market) [8] [9]. The overall size of the sensors are $1 \times 1 \mathrm{~mm}^{2}$ and $0.5 \times 0.5 \mathrm{~mm}^{2}$ for the STMicroelectronics.

In order to characterize the dark-count of the devices, we measured the number of noise pulses generated per unit time as a function of the discriminator threshold. The normalized dark noise for all devices is showed in Figure 4. In the lower left part of same plot is showed the noise level expected in case of no cross-talk in correspondence of the arrow. The plot show that the count rate of the STM device has an effective drop of the curve, while for the other SiPM it still remains high, mainly as a consequence of the cross-talk effect.

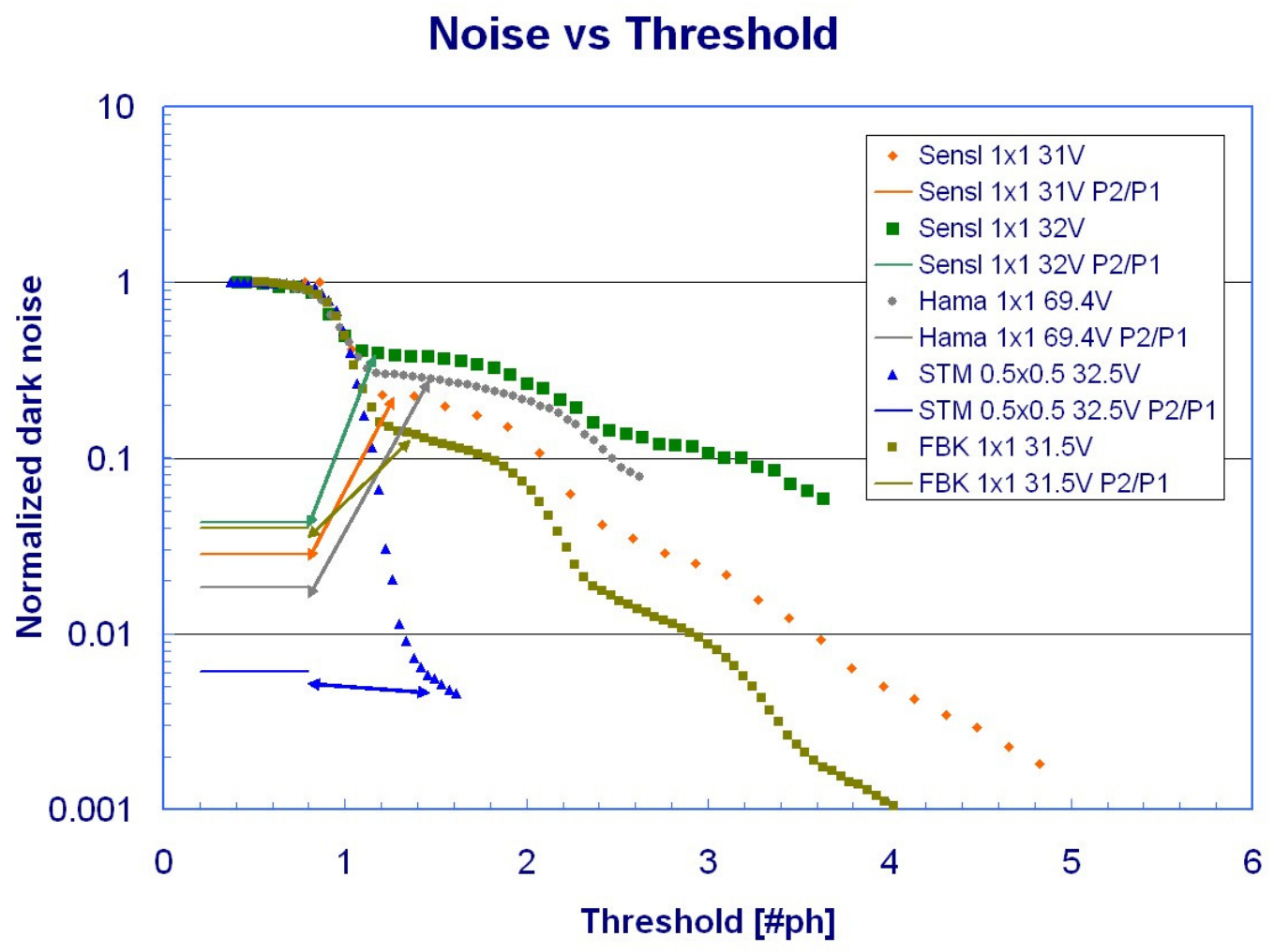

Figure.4 Dark noise as a function of threshold for four different SiPM.

To perform the device characterization to the dark-count and the response to a light of the SiPM's, given their low gain, in a first time we have used a commercial amplifier manufactured by Ortec, (FTA 810b), with a nominal gain of 200 and $400 \mathrm{MHz}$ bandwidth. But for our intentions to implement in a board the whole front-end electronics featuring bias supply, amplifiers, discriminators and coincidence units, has been developed from our electronic 
department, a new amplifier. The main characteristic of the amplifier are a nominal gain of 200 with a bandwidth in the range $1 \mathrm{MHz}-4 \mathrm{GHz}$. The best performance of the amplifier are evident in the below figure 5 .
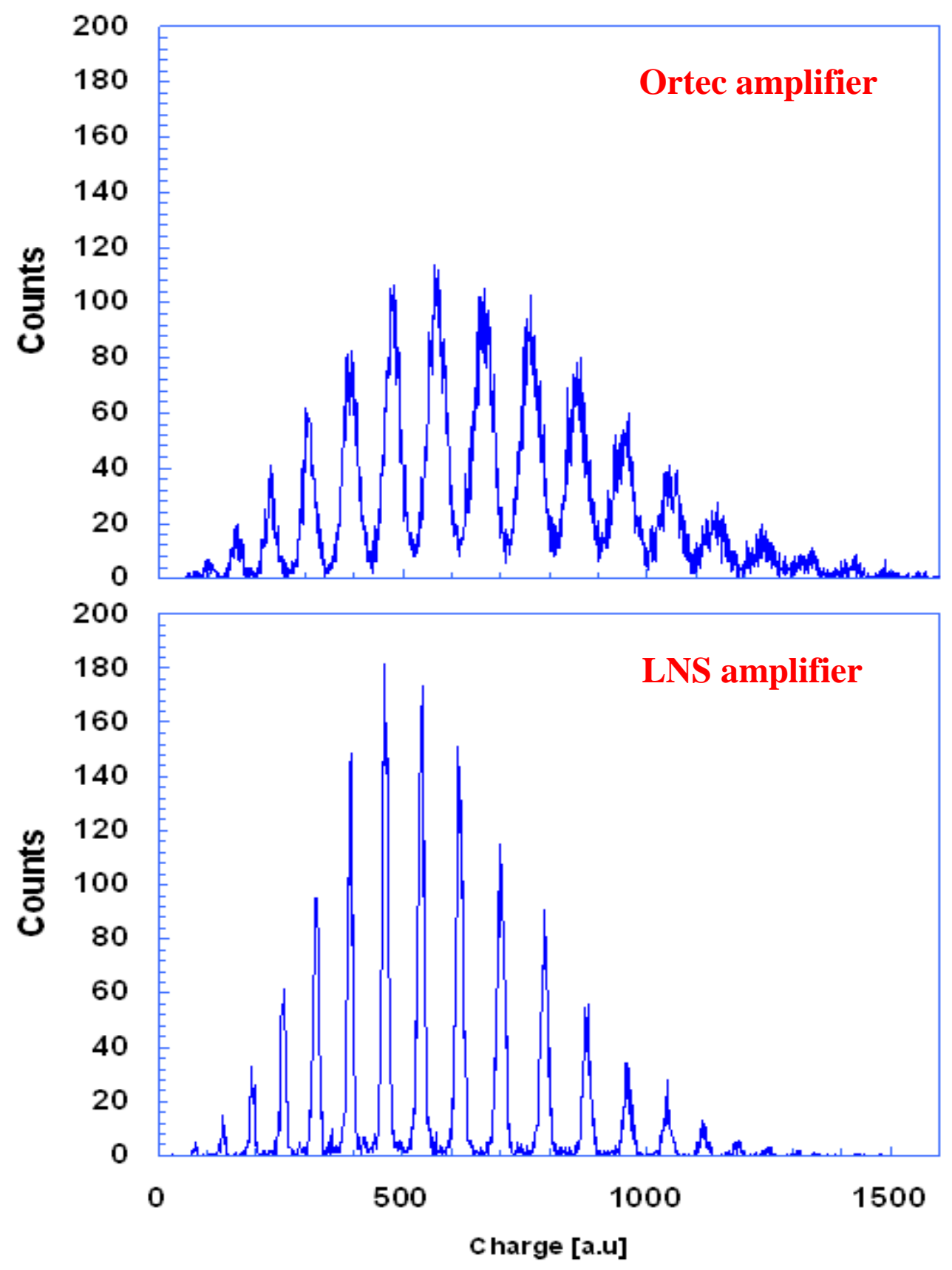

Figure.5 Charge spectrum of a SiPM (10x10 cells, produced by ST Microelectronics) illuminated by means of a pulsed laser, taken using the ORTEC amplifier (a) and the new one developed at INFNLNS (b). The 3-sigma peak resolving power is respectively $\approx 20$ and $\approx 115$ [10]. 


\section{The prototype}

We have arranged our first prototype using three fibres arranged in a ring geometry around a drum made from an empty 35-liters plastic bottle. Every fibres was coupled with two SiPM ( $1 \mathrm{~mm} \times 1 \mathrm{~mm}$ by SensL) one at each end of fibre operating in coincidence in order to reduce the spurious counts. For every SiPM was set a threshold corresponding to 5 triggered cells. A box containing three different gamma radioactive sources with a total activity of $2.7 \mathrm{MBq}$, was positioned in two different position inside the drum while recording the counting rates on the three fibres. The plot in Figure 6 show the results obtained, where it is visibly evident that the system arranged is quite sensitive to variations of the radioactivity distribution, in our case produced by the displacement of the source.

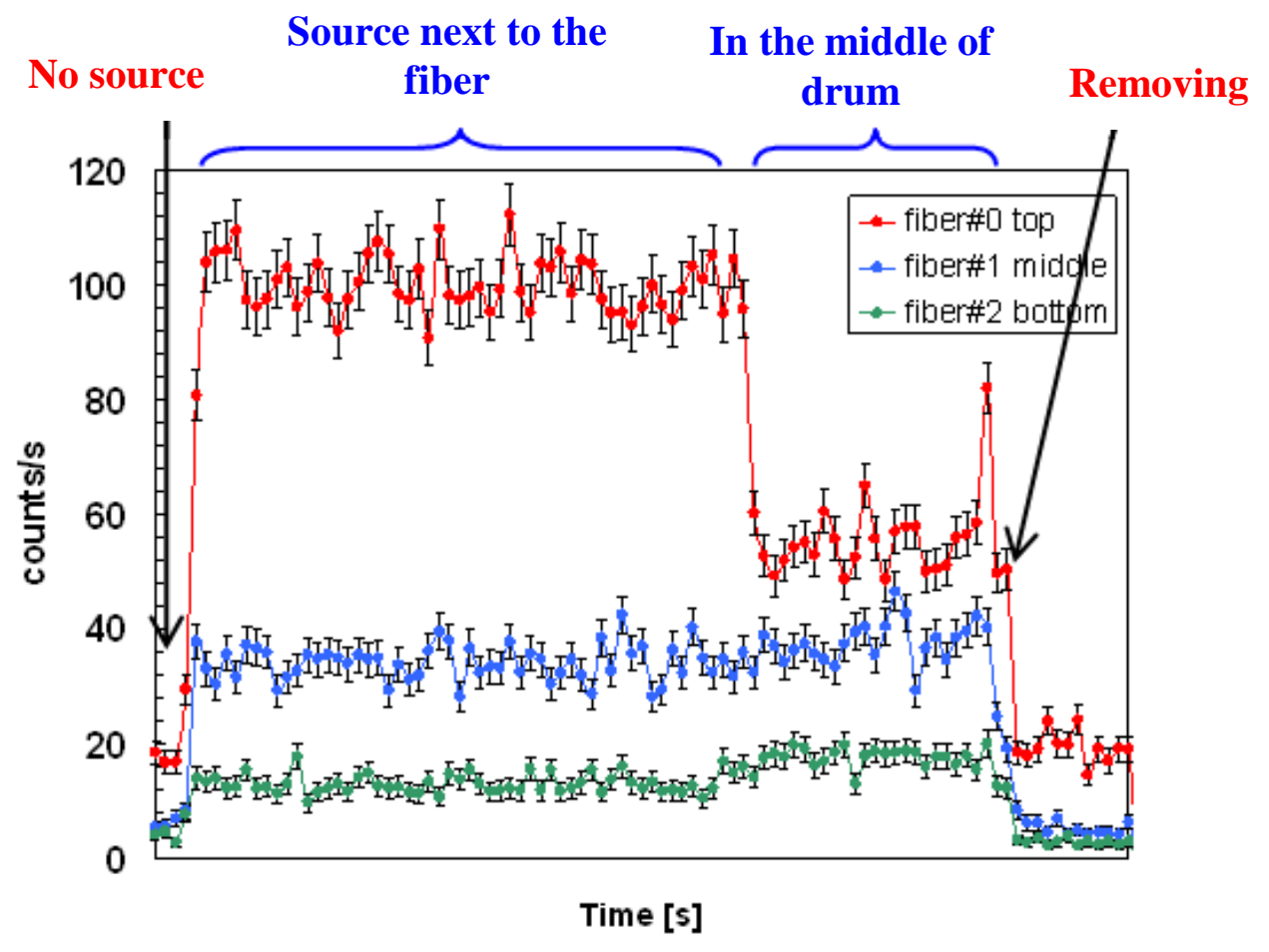

Figure.6: Test results with three fibres around a plastic drum of 35 liters. The counting rate of the three fibres is reported.

\section{Conclusions}

The system has demonstrated an efficient real time monitoring of the radioactive distribution around the drum. The prototype allows to record the measured activity thus providing on line access to the counting rates and also the history on each drum and fibres. 


\section{Acknowledgment}

We are grateful to C.Calì, P.Litrico, S.Marino, G.Passaro, F.Ferrera and B.Trovato of the LNS staff for their invaluable support with electronics, computing and the mechanical workshop.

\section{References}

[1] IAEA, Environmental and Source Monitoring, Safety Guide, No. RS-G-1.8.

[2] ANDRA (http://www.andra.fr); ONDRAF/Belgoprocess, Belgium (http://www.nirond.be/); UKAEA (http://www.ukaea.org.uk/).

[3] Reference gamma-ray and waste drum data provided by Ansaldo Nucleare S.p.A., corso Perrone 25, 16161, Genova, Italy

[4] V.Bellini et al., "Implementation of a new low cost detector for low intensity light pulses produced by a radioactive gamma-source" Journal of Applied Radiation and Isotopes, In print and presented at ICRM -2009 Bratislava

[5] Glenn F. Knoll "Radiation Detection and Measurement" chapter 8, pg 251-252

[6] Z. Papandreou et al. NIM A 596(2008)338-346

[7] Carsten Patrick Achenbach. "Active optical fibres in modern particle physics experiments"

[8] E. Sciacca et al., E. Sciacca et al., "Silicon planar technology for single-photon optical detectors", IEEE Trans. Electron. Dev. 50 (2003) p. 918.

[9] P.Finocchiaro et al., "Characterization of a Novel 100-Channel Silicon Photomultiplier-Part I: Noise”, IEEE Trans. on Elect. Dev., Vol.55, No.10, (2008) 2757; Photomultiplier-Part II: Charge and Time”, IEEE Trans. on Elect. Dev., Vol.55, No.10 (2008) 2765.

[10] P. Finocchiaro et al., "Features of Silicon Photo Multipliers: precision measurements of noise, cross-talk, afterpulsing, detection efficiency", IEEE Trans. on Nucl. Science, v56, n3 (2009) 10331041. 